\title{
Procesamiento de información y conformidad
}

\section{Armando Rodríguez Pérez}

\author{
Universidad de La Laguna
}

El conjunto de datos sobre influencia social en situaciones de conformidad, obtenidos en laboratorio, establece que cuando se presenta a los sujetos estímulos ambiguos, el efecto de la presión de grupo es casi total. Los estudios referidos al efecto de la ambigüedad se remontan a la década de los treinta y especialmente al experimento de Sherif (1935) con el efecto autocinético. En estas situaciones los sujetos carecían de marcos de referencia con los cuales contrastar la exactitud de sus respuestas.

Otras investigaciones de esa época apoyaban la idea de esos puntos de comparación y el papel jugado por los factores sociales en las opiniones de los sujetos cuando los estímulos carecían de estabilidad y claridad (Sherif y Cantril, 1945; Festinger, 1954), lo que condujo al establecimiento de diferencias entre la utilización de estímulos ambiguos y no ambiguos. De este último tipo son los estímulos utilizados por Asch (1952).

Esta polémica sobre la ambigüedad del estímulo y el consiguiente papel jugado por otros factores sociales condujo en los años 50 y 60 a una acumulación sorprendente de datos experimentales cuyas explicaciones carecen de la coherencia teórica esperada.

Uno de los temas en los que inciden todos estos estudios es el de la polémica inconclusa sobre difcrencias sexuales $\mathrm{e}$ influenciabilidad. Mientras para muchos autores se está tratando con diferencias de personalidad, diferencias en comportamientos debido al aprendizaje de roles sexuales prescritos, para otros se trata de un problema cuyo centro radica en el procedimiento experimental y, especialmente, en el tipo de estímulos utilizados.

Aunque no es objeto de este estudio el centrarnos en esas diferencias, sí nos puede servir como hilo de la exposición que permita sacar a la luz determinadas inconsistencias en las investigaciones sobre conformidad.

Eagly (1978) proporciona datos relativos a una revisión de 61 estudios que se suelen utilizar como fuente de esas diferencias. Dejando al margen el poco rigor científico que demuestran muchos autores, que no encontrando diferencias significativas (el $62 \%$ ) las defienden como tales, esta autora encontró que en el 32 por 100 de los estudios se obtenía una mayor conformidad en las mujeres, 
mientras en el 3 por 100 se encontraba en los hombres.

Según Eagly (1978) una razón poderosa de estos resultados se debe a los estímulos utilizados y cita algunos de los estudios realizados desde una perspectiva diferente (Sistrunck y McDavid, 1.971; Goldberg, 1974, 1975).

Estos últimos autores, clasificaron los estímulos según el grado de interés que poseía para cada sexo, en items masculinos, femeninos, y neutros, hallando una mayor influenciabilidad en las mujeres ante items masculinos y mayor en los hombres en items femeninos, no encontrando diferencias significativas en los items neutros. Resultados semejantes obtuvo Javorniski (1979).

Eagly (1978) por su parte generaliza estas conclusiones a todos los estímulos utilizados en los experimentos de conformidad, especialmente a los perceptivos que han sido los más numerosos. Según ella, estímulos como los de Asch favorecen la conformidad de las mujeres, debido a la superioridad de los hombres en habilidades visoespaciales y numéricas.

Esta característica, que según la psicología social es explicada por las particularidades del rol sexual $y$, por tanto, de su subsiguiente familiaridad, no concuerda con otros enfoques para los que la familiaridad, aunque significativa, no determina enteramente la certidumbre de los juicios del sujeto frente a los intentos de influencia de un grupo. Según nuestro punto de vista, esto estaría más relacionado con los niveles de análisis a que han sometido los sujetos esos mismos estímulos en su vida pasada, el contexto con que se ha relacionado, y la forma en que se los utiliza para un mejor ajuste al medio.

En este sentido, tanto la observación casual como un extenso cuerpo de investigaciones han comprobado la tendencia que poseen los humanos a organizar los estímulos en categorías que se mantienen almacenadas en la memoria semántica (Tulving, 1972).

La literatura cognitiva sobre la percepción ha producido recientemente un excelente material (Rosch, 1973, 1975 a, 1975 b, 1977; Rips, Shoben y Smith, 1973) sobre la estructura y naturaleza de las categorías naturales, rompiendo, de algún modo, con el punto de vista tradicional según el cual todos los miembros categoriales poseen ciertas características críticas que obligan a tratar los estímulos como pertenecientes o no a una determinada categoría, cuyos límites están perfectamente claros: Para estos autores, las categorías naturales se organizan formando un continuo desde los miembros más prototípicos de una categoría (los que representan mejor la imagen de esa categoría) hasta los menos prototípicos. De lo que se deduce que no todos los elementos de una categoría son equivalentes. Por lo general, los mejores ejemplos de una categoría, sirven como punto de referencia al resto de los miembros categoriales (Rosch, 1975 b), por lo que la tipicalidad no es un producto directo de la familiaridad, sino, más bien, de las definiciones que la sociedad, a través de la cultura y el lenguaje, impone a esos conceptos, proporcionando así una dimensión operativa sobre la que actuará esa familiaridad.

Según esto, un factor que suponemos afectaría a las diferencias en influenciabilidad es justamente esta organización categorial, en la que la ambigüedad apenas estaría relacionada con la dificultad o la poca importancia, sino con la relevancia preceptiva que ese estímulo tiene para el sujeto.

El objeto del presente estudio es justamente comprobar que la frecuencia con que cambia una opinión como resultado de recibir una presión social unánime, varía con la tipicalidad del estímulo utili- 
zado, obteniéndose la máxima influenciabilidad en los puntos más bajos y la mínima en los puntos más altos de esa escala de tipicalidad.

Con este fin, se llevaron a cabo cuatro investigaciones: la primera, exploratoria, tenía por objetivo determinar todos los items que formaban parte de las categorías semánticas en nuestro habla. La segunda, buscaba ordenar esos items de mayor a menor tipicalidad; la tercera, pretendía comprobar por medios experimentales la validez de los resultados anteriores y la cuarta iba destinada a comprobar si nuestros supuestos poseían o no la evidencia empírica suficiente en situaciones de conformidad.

\section{INVESTIGACION 1 Y 2}

Para poder llevar a cabo nuestra investigación sobre los puntos categoriales más sensibles a las presiones grupales fue necesario componer la estructura interna de ciertas categorías semánticas y comprobar a continuación, la fiabilidad de los juicios dada por los sujetos respecto a su estructura interna.

El procedimiento ideado por Rosch (1975), partía de la presentación de listas compuestas por los miembros de cada categoría a las que los sujetos debían dar puntuaciones según su tipicalidad o grado con que representaban la idea que tenían de esa categoría. Sin embargo, la ausencia en nuestra lengua de estudios sistemáticos y fiables sobre categorías semánticas condujo a la realización de una investigación exploratoria, que basándose en la técnica de asociación libre configurara cada categoría. Esta técnica se apoyó en la noción de valor asociativo (Glaze, 1928) y de significado (Noble, 1945) definidos como el promedio de asociaciones continuas dadas a un ítem en un período de tiempo estándar por un grupo de sujetos.
De ser válida esta técnica, la ordenación de cada categoría, en base a la frecuencia con que eran citados los miembros, debería correlacionarse significativamente con la técnica de Rosch (1975), en la que los sujetos debían puntuar en qué medida el título de una categoría sustentaba la imagen de cada miembro.

\section{METODO}

Participaron en el estudio exploratorio 119 sujetos de ambos sexos, estudiantes de $4 .^{\circ}$ y $50^{\circ}$ de psicología. En el segundo estudio participaron 189 sujetos pertenecientes a $30^{\circ}$ de BUP, COU, $1 .^{\circ}$ y 2.0 de Filosofía y Letras. Todos los sujetos respondieron a las pruebas voluntariamente.

\section{PROCEDIMIENTO}

En ambas investigaciones se utilizaron diez categorías concretas (Arma, Ave, Deporte, Flor, Fruta, Herramienta, Mamífero, Mueble, Ropa y Vehículo).

En la primera, se solicitaba a los sujetos, después de unas instrucciones referidas a la categorización humana, la enunciación de todos los miembros de esa categoría que se les ocurriese, y lo más rápidamente posible. $\mathrm{El}$ tiempo para cada categoría fue de 30 segundos.

Una vez obtenidos los miembros de las categorías citados, se dispusieron en folios independientes, formando un cuadernillo, cuyas hojas fueron ordenadas aleatoriamente (diez órdenes diferentes). A su vez, los items dentro de cada categoría, fueron aleatorizados y presentados a la mitad de los sujetos (90) en el orden A y a la otra mitad (92) en el orden inverso $\mathrm{B}$.

Todos los miembros de una categoría se hallaban enumerados bajo el título de 
la categoría. Se les pedía a los sujetos que puntuaran del 1 al 7 en qué medida consideraban representativos cada uno de los ítems respecto a la categoría citada.

Las instrucciones recogían varios ejemplos con el fin de que los sujetos entendieran bien la tarea ( $«$ Piensen por ejemplo en perro. Todos ustedes tienen noción de lo que es realmente un perro. Para nosotros, acordarnos de un perro pastor alemán es pensar en un perro muy perro, mientras que un chihuahua sería un perro menos perro»).

\section{RESULTADOS Y DISCUSION}

Se obtuvieron las $\overline{\mathrm{X}}$ y D.T. de los 489 items, tanto para el total de varones como de hembras, para los sujetos de formato $\mathrm{A}$ y de formato $\mathrm{B}$ y por último, la media total de la muestra.

Posteriormente, se realizaron los cálculos necesarios para comprobar si existían diferencias significativas en la puntuación de tipicalidad dada por los varones, hembras, sujetos con formato $A, y$ con formato $B$.

También se contrastó la ordenación de la primera investigación con los resultados obtenidos en esta segunda fase.

Los coeficientes de correlación entre las dos mitades de la muestra con diferentes formatos, estuvieron entre $r=0.89$ y $r=0.97(p<0.001)$ y entre varones y hembras entre $r=0.87$ y $r=0.96(p<$ 0.001).

La relación entre la ordenación obtenida por medio de la técnica de la asociación libre y la escala de puntuación estuvo para todas las categorías entre $\mathrm{r}=0.56$ y $\mathrm{r}=0.88(\mathrm{p}<0.001)$.

La prueba «t» aplicada a los 489 items dio resultados que merecen una investigación más exhaustiva, sobre todo porque, aunque no en muchos, sí al me- nos en el 30 por 100 de los items de cada categoría, se encuentran diferencias significativas entre varones y hembras, cuando esto sólo ocurre en un 6 por 100 de las puntuaciones según los dos formatos.

Esto podría indicar que la variable sexo contribuye de alguna forma a distinciones en la organización categorial de los estímulos, lo que constituye un hecho de incalculable importancia en nuestros supuestos sobre las diferencias en influenciabilidad debidas a los estímulos.

Por otra parte, los resultados han constatado que existe una fuerte similaridad en las distintas formas en que los sujetos han informado acerca de las categorías semánticas, comprobándose en ambos casos la utilidad de la técnica y el acierto de nuestras suposiciones.

\section{INVESTIGACION III}

Las investigaciones anteriores apuntan que la pertenencia de un miembro a una categoría es una cuestión de grado, donde los más típicos son mejores ejemplos que los menos típicos.

Rosch (1975 a), partiendo de esa misma opinión, demostró que las puntuaciones dadas por los sujetos eran predictivas del tiempo de reacción necesitado por éstos en tareas de verificación de sentencias del estilo A (miembro de la categoría) es B (categoría). Rips, Shoben y Smith (1973) y Smith, Shoben y Rips (1974) llegarón a conclusiones semejantes, apuntando que los modeios explicativos que estaban tras los datos podían explicar muchos resultados sobre investigaciones referidas a la recuperación de información en la memoria semántica.

Sobre esta base, el objetivo de esta investigación era comprobar si los TR de los items variaban según la puntuación 
de tipicalidad dada por los sujetos, configurando una tendencia lineal significativa.

\section{METODO}

\section{Sujetos}

Participaron en este experimento 32 sujetos, de los cuales 16 eran varones y 16 hembras.

\section{Estímulos}

De las 10 categorías inicialmente investigadas, escogimos las siete que obtuvieron correlaciones más altas entre la técnica de asociación libre y la escala de puntuación (aun cuando las diferencias no fueron amplias).

De cada una de esas siete categorías (Fruta, Ave, Deporte, Arma, Mueble, Flor y Mamífero) se escogieron ocho miembros, que se distribuyeron a lo largo de la escala. Para ello, se dividieron las puntuaciones de tipicalidad dadas por los sujetos en ocho intervalos y, de cada uno de ellos, se escogió aleatoriamente un miembro, rechazando aquellos casos en los que el item seleccionado tuviera puntuaciones significativamente diferentes, según el sexo de los respondientes.

En total, se trabajó con 57 items experimentales, a los que se añadieron 14 , cuya falsedad evitaría la habituación del sujeto a dar respuestas afirmativas. Así, en los items experimentales se presentaba un miembro y su categoría (por ejemplo, la rosa es una flor), mientras en los items de control era evidente la falsedad de la sentencia (por ejemplo, la gallina es una flor). El orden de los estímulos fue aleatorio y fueron presentados a los sujetos en dos formatos: el aleatorio y el inverso.

Todos los estímulos se presentaron a través de taquistoscopio y los tiempos de reacción fueron obtenidos a partir de un timer conectado a aquél.

\section{Procedimiento}

Una vez en el laboratorio, el sujeto atendía a las instrucciones, que consistieron principalmente en:

«... A continuación se le presentará en el taquistoscopio un conjunto de proposiciones en forma afirmativa.

Usted puede o no estar de acuerdo con estas proposiciones. Vea que delante de usted hay un interruptor.

Cuando sepa con seguridad que esta proposición es correcta, o que se ajusta a su opinión, debe apretarlo inmediatamente, al tiempo que dice en voz alta ¡Sí!

El tiempo de duración del experimento osciló entre 15 y 20 minutos, aproximadamente.

\section{Resultados}

La media total de los TR de cada miembro en cada una de las categorías correlacionó con el rango de las puntuaciones en la escala de puntuación, por encima de $r=0.74(p<0.001)$.

Los análisis de varianza llevados a cabo, con el fin de comprobar si realmente existían diferencias significativas en los tiempos de reacción de los miembros de cada categoría, dieron $\mathrm{F}$ en todos los casos superiores a $19.6(\mathrm{p}<0.001)$

Para cuatro de esas categorías, más del 80 por 100 de la varianza es expli. cada como tendencia lineal, mientras que para el resto esta tendencia explica, al menos, el 40 por 100 .

\section{Discusión}

Los resultados apoyan nuestras hipó tesis iniciales, a la vez que concuerdan 
con los estudios precedentes, en el sentido de que las puntuaciones de los sujetos fueron buenas predictoras de los tiempos de reacción de éstos ante los items categoriales.

En ese mismo camino, el análisis de tendencia permitió comprobar que, aunque imperfectamente, predomina una linealidad en la distribución del tiempo de reacción a lo largo de la jerarquía categorial. También las desviaciones típicas muestran un progresivo aumento a medida que descendemos en la puntuación de tipicalidad.

Menos explicables desde nuestra perspectiva son las diferencias en los tiempos de reacción de los elementos más típicos de una categoría a otra. Así, mientras varios de nuestros ejemplos se ajustan más o menos a un tiempo que oscila entre 1.24 y 1.30 para el item más típico (por ejemplo: paloma 1.24; rosa 1.25 ; naranja 1.27 ; fútbol 1.28 ; mesa 1.30 ), otros se salen claramente de este margen (pistola $1.38 \mathrm{y}$, sobre todo, hombre 1.61).

Algo similar ocurre en los items menos típicos de las diferentes categorías, cuyas fluctuaciones van desde 2.65 hasta 4.02 .

\section{INVESTIGACION 4}

En este punto, nuestro objetivo reside en averiguar si la influenciabilidad varía según el punto de la escala de tipicalidad sobre el que se actúa, o si, por el contrario, depende de factores que hasta ahora han servido para explicar la modificación de la opinión de los sujetos. Nos referimos a la dificultad, ambigüedad perceptiva del estímulo, falta de importancia, experiencias previas en tareas semejantes, etc....

Con este fin se llevó a cabo un experimento en el que paralelamente a la manipulación de la tipicalidad categorial, se manipuló la información que los sujetos experimentales tenían sobre el grupo de presión, que en nuestro caso era ficticio, ya que se trabajaba con aparatos semejantes a los utilizados por Crutchfield (1955).

El tipo de información presentada hacía referencia a la mayor familiaridad del grupo en tareas semejantes, la identidad nacional del grupo con los sujetos expe. rimentales, y la similaridad actitudinal entre el grupo y los sujetos experimentales.

Estos tres aspectos, a pesar de constituir, desde una óptica reduccionista, un resumen de las características más comúnmente atribuidas a los emisores, no han sido objeto de estudio en situaciones de conformidad más que de forma aislada y con resultados no demasiado fiables (Warren, 1969; Leet-Pellegrini y Rubin, 1974; Peay, 1976).

Nuestros supuestos se apoyan en tres ideas básicas: Primero, es más probable lograr influencia en la zona de menor tipicalidad de la escala categorial. Segundo: Esto se logrará independientemente de las categorías utilizadas, $\mathrm{y}$, tercero: Ambas suposiciones se mantendrán constantes en todas las situaciones experimentales, independientemente de la información que se proporcione a los sujetos experimentales sobre el grupo de presión.

\section{METODO}

\section{Sujetos}

Formaron parte de esta investigación 270 sujetos de ambos sexos, alumnos de psicología y pedagogía, cuya edad era inferior a los 26 años y no habían participado en ninguna de las investigaciones anteriores. Treinta de estos sujetos fueron utilizados para entrenar a los expe- 
rimentadores y sus resultados no se tuvieron en cuenta.

Los 240 restantes fueron distribuidos aleatoriamente en los cinco grupos experimentales.

\section{Estímulos}

Se utilizaron como estímulos los miembros de tres de las categorias previamente investigadas (Mueble, Ave, Flor).

Los ocho miembros obtenidos en la investigación 3 se emparejaron según el criterio de distancia intracategorial, agrupándolos posteriormente en tres bloques: Uno de distancia Larga (DL), con parejas cuyos valores son: 1-6, 2-7, 3-8; otro de distancia corta (DCS), en la mitad superior de la categoría (parejas en posiciones $1-2,2-3,3-4) y$, por último, el de distancia corta en la pareja inferior (DCI), que recogía los pares 5-6, 6-7 y $7-8$.

Siguiendo el procedimiento experimental de Asch (1952), incluimos items que desempeñarán la función de estímulos creibles $y$, por tanto, carentes de todo valor experimental.

La utilización de tres categorías se debía fundamentalmente a nuestra sospecha de que la presentación iterativa de un estímulo (si presentamos los 9 pares de una sola categoría se repetirán dos veces los que ocupan la posición 1 y 8 y tres veces los que ocupan la 2, 3, 6 y 7) podría cambiar «momentáneamente» por su familiarización, el valor de tipicalidad de esos miembros en la escala categorial.

Utilizando tres categorías equivalentes, cada sujeto recibía una combinación de ellas, de forma que nunca se repitiera un mismo miembro. Estas combinaciones dieron lugar a tres series en las que se balanceó el orden de presentación de cada item de la pareja.

El laboratorio estaba constituido por cinco cabinas, una de las cuales estaba ocupada por un experimentador que manipulaba las respuestas que aparecían en los aparatos de los sujetos y recogía a su vez las respuestas que daban a cada estímulo.

Antes de su entrada en el laboratorio, los sujetos pasaban una entrevista que confirmaba a los investigadores que ese sujeto no había oído ningún tipo de comentario sobre la investigación. Estas entrevistas las conducían cuatro colaboradores del investigador que actuaban simultáneamente, acompañando a los sujetos en momentos diferentes al laboratorio, con el fin de que nunca llegaran a saber quienes estaban en las otras cabinas.

Al terminar la sesión experimental los sujetos eran nuevamente entrevistados por estos colaboradores $y$ respondían a un cuestionario que medía la efectividad de la instrumentación.

Las instrucciones experimentales variaban para las cinco condiciones. En el grupo control (GC) los sujetos debían responder cual era el ítem de cada pareja que consideraban más representativo.

En el grupo sin información (SI) los sujetos eran advertidos de que ocupaban el orden número 5 y que, por tanto, conocerían a través del aparato las respuestas de los otros sujetos.

En los grupos de experiencia previa (EP), idéntico origen nacional (canarios) (IC) y similaridad de actitudes (SA), recibían además de las instrucciones, informaciones detalladas sobre los otros (informaciones ficticias e idénticas para todos) y sobre sí mismos (se poseía bastante información de los sujetos a esos tres niveles).

Las respuestas del grupo ficticio (dadas por el experimentador) a los estímulos era en todos los casos unánimes y en favor del elemento menos típico. Puesto que la tarea era determinar el miembro más típico, la situación experimental 
planteaba a los sujetos un conflicto entre lo que ellos realmente consideraban y la opinión «mayoritaria», que iba en sentido opuesto.

Se utilizaron las respuestas del grupo de control para verificar las respuestas que los sujetos darían sin el grupo de presión.

\section{Resultados}

Se llevó a cabo un ANOVA de $3 \times 3 \times$ 5 con medidas repetidas en los dos pri- meros factores. Como variable dependiente se consideró el número de personas de cada ocho que respondían como el grupo de presión (para los cuatro grupos experimentales) o de forma contraria a como esperábamos sin actuar esa presión (en el grupo de control). Esta agrupación se hizo, fundamentalmente, con el fin de obtener una escala de medida de. razón que permitiera la realización de un análisis de varianza.

En la tabla 3 se presentan los resultados.

\section{Fuente de varianza}

Tipo de información sobre

el grupo presión

Error $\times T$

Distancia (D)

$\mathrm{T} \times \mathrm{D}$

Error $\times \mathrm{D}$

Categorias

$\mathrm{T} \times \mathrm{C}$

Error $\times \mathrm{C}$

$\mathrm{D} \times \mathrm{C}$

$\mathrm{T} \times \mathrm{D} \times \mathrm{C}$
$\mathrm{D} \times \mathrm{C} \times$ Error $\mathrm{D} \times \mathrm{C}$
SC

313.3

47.59

352.02

36.79

72.30

13.09

12.95

65.74

8.76

10.10

106.04 .
GL

$M C$

F

$P<$

Lo más característico de estos resultados es, en primer lugar, el valor de la $\mathrm{F}$ en la variable distancia. Efectivamente, tal y como veníamos argumentándolo, existían fuertes diferencias entre las influencias obtenidas, según el punto de la escala de tipicalidad sobre el que actuáramos. Su acción varía ligeramente $F=2.07(p<0.05)$, según tratemos con una categoría u otra. En nuestro caso concreto, las influencias obtenidas se reducen considerablemente para la categoría flor (Frecuencia total en la categoría flor $=252$ frente a 293 en la categoría mueble y 295 en la categoría Ave), especialmente, en DL y en DCS. De estas diferencias en los totales da buena muestra, la $F=4.98(\mathrm{p}<0.001)$.

En lo que se refiere a las diferencias entre las cinco condiciones experimenta- les hay que remarcar que la mayor parte de esa $F$ da cuenta del contraste con el grupo de control. Un análisis más pormenorizado, siguiendo la técnica de Dunnet (1955), no halló diferencias significativas entre los cuatro grupos experimentales, aunque como se puede constatar en la tabla 3, existe una ligera interacción $(\mathrm{F}=3.17, \mathrm{p}<0,001)$ entre estas condiciones y las distancias utilizadas en los estímulos.

Estas interacciones manifiestan una progresiva disminución de las diferencias de influenciabilidad entre la DCS y la DCI en las condiciones IC, SA, EP, terminando en el grupo SI en los que se consiguen prácticamente el mismo número total de cambios (DCS $=89$; $\mathrm{DCI}=88$ ).

Estos resultados respondieron total- 
mente a nuestras previsiones, sobre todo en lo que se refiere a las diferencias según las posiciones de la categoría en las que actuáramos. Así, el cambio inducido es mayor en los elementos menos típicos de una categoría que en los más típicos, y menor cuando se intenta intercambiar items típicos por menos típicos.

\section{Discusión general}

Los resultados de este trabajo lograron claramente apoyar nuestros supuestos iniciales, según los cuales el término ambigüedad podia asimilarse al nivel en que cada ítem se encuentre organizado en la memoria semántica.

Este estudio ha partido de pautas concretas en la categorización de la información y la utilización del producto final como un factor explicativo de las inconsistencias halladas en torno al estudio de la conformidad individual, que han buscado la clave en aspectos que no se apartaban totalmente de lo aquí defendido.

Nuestro estudio pretende salvar la tendencia a utilizar sólo las características situacionales o sólo los rasgos de personalidad en la explicación de un fenómeno en el que, así lo hemos considerado, habría que hacer uso de los paradigmas actuales del procesamiento de información.

Sobre esta base citamos los estudios empeñados en encontrar diferencias sexuales en irfluenciabilidad, al margen de cualquier preocupación por los estímulos, cuando tanto en las nuevas orientaciones marcadas por Sistruck y McDavid (1971) y por Eagly, como por los estudios de categorización, se han hallado diferencias de base que sesgan, sin duda, los resultados experimentales.

Por otro lado, es coherente con nues- tros resultados la resistencia al cambio sobre todo en las distancias largas, dada la dificultad de conseguir intercambio entre lo que constituye un punto de referencia categorial (miembros prototípicos) y un punto cualquiera del contexto, lo que coincide con los resultados obtenidos por la mayor parte de los autores citados en este trabajo.

Así, por ejemplo, en el estudio de Luchins (1945), en el que se presentaban items perceptivos que representaban una botella o un paisaje con láminas intermedias en las que la botella iba desfigurándose y convirtiéndose en el paisaje, los resultados indicaron una mayor conformidad en las respuestas a los items intermedios que a los items extremos, confirmando la idea de que a medida que se da un mayor acercamiento de los estímulos a los puntos de referencias mayor tiende a ser la resistencia del sujeto a las opiniones de los demás, se presente o no en forma unánime.

También nuestras conclusiones concuerdan con los estudios de Tajfel sobre categorización $(1969,1972)$ en los que se tiene en cuenta el background sociocultural del sujeto. Así, determinados valores sociales imprimen una marca a las informaciones procesadas por el individuo. Estas informaciones, consonantes con los valores, son a su vez tanto o más resistentes al cambio que los mismos valores sociales.

Quedaría por saber si este tipo de situaciones suponen realmente una modificación en el punto de vista del sujeto o tan sólo una forma de evitar el conflicto que le proporcione el sentirse en minoría en una situación experimental, que por las características de nuestros sujetos (fundamentalmente de $10^{\circ}$ de psicología y magisterio) era predominantemente ingenua. 


\section{Resumen}

Mientras ciertos estudios llevados a cabo por psicólogos sociales ban comprobado que la conformidad experimental es en gran medida un producto del tipo de estimulos utilizados y de la familiaridad de los sujetos con la tarea, los psicólogos cognitivos ban descubierto que las categorías poseen una estructura interna, cuya organización depende del grado con que cada elemento representa la imagen que tenemos de esa categoria (tipicalidad).

Estos datos dieron lugar a la bipótesis de que la conformidad es función del nivel de tipicalidad de los estímulos, obteniéndose la máxima puntuación en los menos típicos y la mínima en los prototípicos.

Se llevaron a cabo cuatro investigaciones, las tres primeras destinadas a constituir los miembros categoriales y su ordenación, según la tipicalidad. La cuarta manipuló el contenido de las categorias semánticas (ave, flor y mueble), el valor de tipicalidad de los items (tres distancias internas), y la información que los sujetos experimentales tenian del grupo de presión ficticio (control, sin información, experiencia previa, identidad nacional $y$ semejanza actitudinal).

Los resultados apoyaron la relación entre conformidad y tipicalidad, independientemente de la acción de las otras variables manipuladas.

\section{Summary}

Whereas several studies carried out by social psychologists bave demonstrated that the experimental conformity is, to a great degree, a product of the type of stimulus used and the familiarity of the subjects witb the task, cognitive psycbologists bave found that categories bave an internal structure, whose organizations depends upon the degree with wich every element represents the image we bave of that category.

These data had given rise to the bypothesis that the conformity is a function of the level of stimulus typicality, the bigher score is obtained with the less typical and the lower. score with the prototypical.

Four investigations were carried out, the first three were utilized to obtain the category members and its ranking, according to typicality. The fourth investigation manipulated the content of the semantic categories (bird, flower and furniture), the typicality value of the items (three internal distances), and the information that experimental subjects had about the fictious pressure group (control, no information, previous experience, national identity and attitude similarity).

The results lended support to the relation between conformity and typicality, inaffected by the influence of the other manipulated variables.

\section{Referencias}

Asch, S., Social psycbology, Prentice-Hall, 1952 (traducción cast. en Eudeba).

CRUTCHFIElD, R., «Conformity and character», American Psychologist, 1955, 10, 191-198.

DUNNET, $C$., «A multiple comparison procedure for comparing several treatments with a control», Journal of American Statistical Association, 1955, 50, 1096-1124.

EAGLY, A., «Sex differences in influenciability», Psycbological Bulletin, 1978, 85, 86-116.

FESTINGER, L., "A theory of social comparison process», Human Relation, 1954, 7, 117-140.

GLAZE, J., "The associate value of non sense syllabe», Journal of Genetic Psycbology, 1928, $35,255-267$.

GolDBERG, C., «Sex Roles, task competence and conformity», Journal of Psychology, 1974, 86, 157-164. 


\section{Estudios}

GOLDBERG, C., «Conformity to majority type as a function of task and acceptance of sex-related stereotypes», Journal of Psychology, 1975, 89, 25-37.

JAVORNISKY, G., "Task content and sex differences in conformity», Journal of Social Psycbology, $1979,108,213-220$.

LeET-Pellegrin, H. y RuBin, J., «The effects of six bases of power upon compliance, identification and internalizationm, Bulletin Psychonomie Society, 1974, 3, 68-70.

Luchins, A., "Social influences on perception of complex drawings», Journal of Social Psychology, 1945, 21, 257.273.

NoBle, C., «An analysis of meaning», Psychological Review, 1952, 59, 421-430.

PEaY, M., "The effects of social power and preexisting attitudes on public and private responses to and induced attituden, Human Relations, 1976, 29, 1115-1129.

Rips, L.; ShOBen, E., y SMITh, E., «Semantic distance and the verification of semantic relations", Journal of verbal Learning and verbal Bebavior, 1973, 12, 1-20.

Rosch, E., «Natural Categories», Cognitive Psychology, 1973, 4, 328-350.

Rosch, E., "Cognitive representations of semantic categories», Journal of experimental Psychology: General, 1975 (a), 104, 192-233.

Rosch, E., «Cognitive reference points», Cognitive psycbology, 1975 (b), 7, 532-547.

Rosch, E., "Classification of real world object», en Johnson-Laird and W ason (ed.), Thinking: Reading in cognitive science, Cambridge University Press, 1977.

SHERIF, M., «A study of some social factors in perception», Arcbives of psychology, 1935, vol. 27 , núm. 187.

Sherif, M. y Cantril, H., «The psychology of attitudes», Psycbological Review, 1945, 52, 295-319.

SistrunK, F. y McDAvid, J., «Sex variables in conforming behavior», Journal of personality and Social psychology, 1971, 17, 200-207.

Smith, E.; Shoben, E., y RIPS, L., «Structure and process in semantic memory», Psychological Review, 1974, 81, 214-241.

TAJFEL, H., «Social and cultural factors in perception», en Lindzey y Aronson (ed.), The bandbook of social psychology, vol. 3, Addison Wesley, 1969.

TAJFEL, H., «La categorization sociale», en Moscovici (ed.), Introduction a la psycbologie sociale, Ed. Larrouse, 1972. (Traduc. cast. en Planeta.)

Tulving, E., «Episodic and semantic memory», en Tulving y Donalson (ed.), Organization of memory, Academic Press, 1972.

WARREN, D., «The effects of power bases and peer groups on conformity in formal organizations», Administrative Science Quarterly, 1969, 14, 544-546. 